\title{
Vibration Based Diagnosis for Planetary Gearboxes Using an Analytical Model
}

\author{
Liu Hong, ${ }^{1,2}$ Yongzhi Qu, ${ }^{1,2}$ Yuegang Tan, ${ }^{1,2}$ Mingyao Liu, ${ }^{1,2}$ and Zude Zhou ${ }^{1,2}$ \\ ${ }^{1}$ School of Mechanical and Electronic Engineering, Wuhan University of Technology, 122 Luoshi Road, Wuhan 430070, China \\ ${ }^{2}$ Hubei Digital Manufacturing Key Laboratory, Wuhan University of Technology, 122 Luoshi Road, Wuhan 430070, China \\ Correspondence should be addressed to Yongzhi Qu; yongzhiqu@hotmail.com
}

Received 21 July 2016; Revised 4 October 2016; Accepted 26 October 2016

Academic Editor: Ganging Song

Copyright (c) 2016 Liu Hong et al. This is an open access article distributed under the Creative Commons Attribution License, which permits unrestricted use, distribution, and reproduction in any medium, provided the original work is properly cited.

\begin{abstract}
The application of conventional vibration based diagnostic techniques to planetary gearboxes is a challenge because of the complexity of frequency components in the measured spectrum, which is the result of relative motions between the rotary planets and the fixed accelerometer. In practice, since the fault signatures are usually contaminated by noises and vibrations from other mechanical components of gearboxes, the diagnostic efficacy may further deteriorate. Thus, it is essential to develop a novel vibration based scheme to diagnose gear failures for planetary gearboxes. Following a brief literature review, the paper begins with the introduction of an analytical model of planetary gear-sets developed by the authors in previous works, which can predict the distinct behaviors of fault introduced sidebands. This analytical model is easy to implement because the only prerequisite information is the basic geometry of the planetary gear-set. Afterwards, an automated diagnostic scheme is proposed to cope with the challenges associated with the characteristic configuration of planetary gearboxes. The proposed vibration based scheme integrates the analytical model, a denoising algorithm, and frequency domain indicators into one synergistic system for the detection and identification of damaged gear teeth in planetary gearboxes. Its performance is validated with the dynamic simulations and the experimental data from a planetary gearbox test rig.
\end{abstract}

\section{Introduction}

With the advantages of coaxial shafting, compact structure, and high torque to weight ratio, planetary gearboxes have been widely employed in heavy-duty applications such as helicopters, offshore rigs, and wind turbines. Since unexpected failures of gearboxes in heavy-duty drive trains may lead to expensive downtime losses and catastrophic accidents, the condition monitoring of planetary gearbox is essential to reduce the maintenance costs and ensure the safety operations. In industrials, vibration based diagnosis using accelerometers mounted on the gear/bearing housing has prevailed in the recent decades because it does not interfere with the normal operation of rotating machines [1]. The gear failures in fixed-axis gearboxes can be directly detected by observing the presence of sidebands around the gear mesh harmonics in the measured vibration spectrum [2]. However, as planetary gear systems employ $P$ number of identical planet pinions revolving around the sun gear with the carrier, dominant sidebands around gear mesh harmonics are observed even when no gear failure or severe manufacturing error exists $[3,4]$. Thus, the fault detection criteria for planetary gearboxes are not straightforward compared with the fixed-axis gearboxes due to the complexities of sidebands in the vibration spectrum measured by the fixed accelerometer. Existing literatures have also found that the sidebands are often asymmetric and the gear mesh harmonics may not even show up in the measured vibration spectrum for specific gear-set geometries, which are the result of manufacturing errors and the superposition of vibrations from $P$ planets $[5,6]$. Moreover, the noises and vibrations from other stages of planetary gearboxes may further mask the fault signatures of planetary gear-sets, which make the vibration spectrum more difficult to interpret.

To address the relatively complex behaviors of frequency components and the superposition of vibrations from $P$ planets, a vibration separation (VS) methodology was developed for the fault detection of planetary gearboxes in helicopter 
transmissions $[7,8]$. The well-known denosing technique, time synchronous averaging (TSA), was also studied to isolate the vibration of planetary gear-sets from noisy sensory signals, which is equivalent to filter the raw vibration data through a comb filter [9]. However, the applicability of VS and TSA to planetary gearboxes is often limited in practice because they require extensive stationary vibration signals and additional knowledge of the time instant when each planet passes through the fixed accelerometer. Furthermore, in practical industrial applications, the simple detection of gear fault is often insufficient. The fault identification is also of interest as the replacement gears can be ordered before the actual disassembly of gearboxes, which in turn reduces the machine downtime [10]. Thus, to advance the state-of-art of diagnosis of planetary gearboxes, it is critical to develop a novel diagnostic system for industrials that is applicable to intelligently highlight and identify the fault signatures of planetary gear systems from the noisy and profuse measured raw data.

To this aim, a novel synergistic diagnostic scheme is proposed in this work that enables the automated detection and identification of damaged gear teeth for planetary gearsets. The paper begins with an introduction of the analytical model of planetary gear systems developed by the authors [11], which can characterize the influence of different planetary gear faults on the sidebands of vibration spectrum measured by a fixed accelerometer mounted on the annulus gear. The presented analytical model has also been validated by extensive experimental studies in [12]. Then, to address the limitation of the requirement of extensive stationary data of TSA, a novel denoising algorithm for planetary gearsets is proposed herein to highlight the fault introduced sidebands by employing a comb filter in the frequency domain. This fault signature enhanced filter is based on the summarized analytical model instead of the ensemble of the average of numerous revolutions. Afterwards, frequency domain indicators are proposed for the intelligent fault identification. The overall diagnostic scheme integrates the analytical vibration model, a proposed denoising algorithm, and frequency domain indicators into one synergistic system for the automated diagnosis of damaged gear teeth, which is capable of carrying out vibration based condition monitoring of planetary gearboxes. Finally, the effectiveness of the proposed diagnostic scheme is validated using both the simulated data from a dynamic model of planetary gearbox and experimental signals from a $4 \mathrm{~kW}$ planetary gearbox test rig.

\section{Review of the Geometry Based Analytical Model of Planetary Gear Systems}

Figure 1 illustrates a typical configuration of planetary gearsets as an example, which includes $P(P=4)$ identical planet pinions. As a practical wind turbine planetary gearbox, the planet carrier $c$ and the sun gear $s$ are assumed to be connected with the input and output shaft, respectively. The annulus gear $a$ is fixed with the gear housing. $\psi_{i}$ is defined as the initial angular position of the $i$ th planet, in

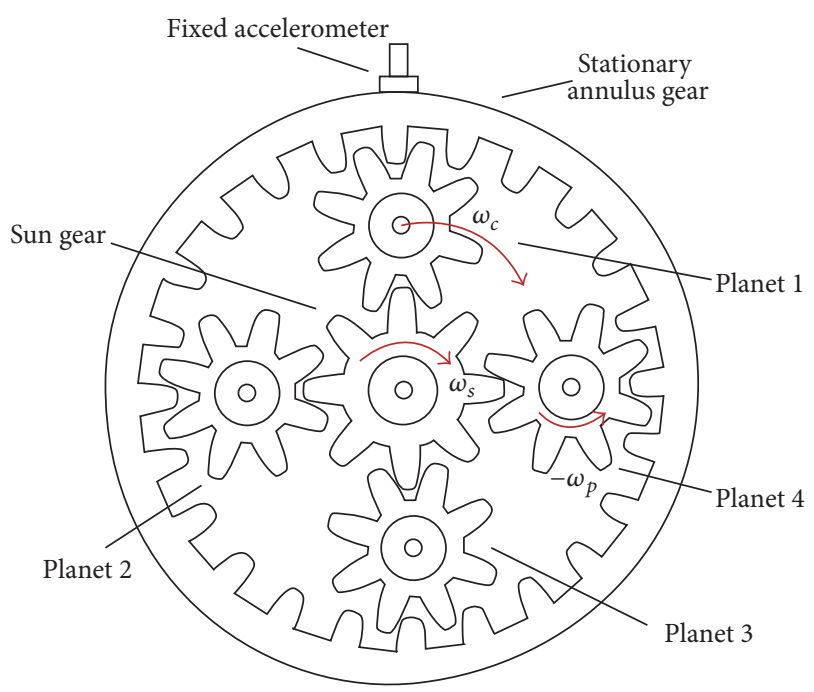

FIGURE 1: A typical configuration of planetary gear-set with 4 equally spaced planet pinions and an accelerometer mounted on the stationary annulus gear.

which $\psi_{i}=2 \pi(i-1) / P$. If planet pinions rotate counterclockwise with an angular rotational frequency $\omega_{p}$ as shown in Figure 1, the sun gear $s$ and the carrier $c$ would rotate clockwise with an angular rotational frequency $\omega_{s}$ and $\omega_{c}$. The angular rotational frequency of the annulus gear is $\omega_{a}=0$. The fundamental gear mesh frequency of the planetary gear system $\omega_{m}$ can be calculated as $\omega_{m}=N_{a} \omega_{c}=N_{s}\left(\omega_{s}-\omega_{c}\right)=$ $N_{p}\left(\omega_{p}+\omega_{c}\right)$, in which $N$ represents the number of teeth on the gear with respect to the subscript.

The vibration signal measured by the accelerometer mounted on the stationary annulus gear contains valuable diagnostic information of the planetary gear-set. This measured vibration signal $z(t)$ by the fixed accelerometer, which is the superposition of vibrations from $P$ planets meshing with the annulus and sun gear, can be expressed as $[11,12]$

$$
z(t)=\sum_{i=1}^{P} w_{i}(t)\left[x_{i}(t)+y_{i}(t)\right],
$$

where the window function $w_{i}(t)$ is used to describe the amplitude modulation phenomenon, which is caused by the relative motion between the fixed accelerometer and the revolving planets with the carrier $[5,6]$. The details can be explained as follows: as a planet moves toward the accelerometer, its vibration dominates the sensory data. The level of its contribution reaches a peak when the planet is almost under the accelerometer and then decreases as the planet recedes from the accelerometer. Thus, the window function $w_{i}(t)$ is periodic with the carrier rotational frequency $\omega_{c}$, the shape of which is usually modeled as either a Hann window or Turkey window [13].

The vibration signal $x_{i}(t)$ in (1) represents the vibration that resulted from the annulus gear and $i$ th planet mesh, which is periodic at the fundamental gear mesh frequency $\omega_{m}$. Note that the vibration waveforms resulting from $P$ different annulus-planet gear meshes are similar but shifted 
in time because of the variation in the initial phase during the gear meshes [6]. Therefore, the vibration signal $x_{i}(t)$ can be written as a Fourier series $[6,11]$ :

$$
\begin{aligned}
x_{i}(t) & =\sum_{j=1}^{J} X_{i j} \cos \left(j N_{a} \omega_{c}\left(t-\frac{\psi_{i}}{\omega_{c}}\right)+\theta_{j}\right) \\
& =\sum_{j=1}^{J} X_{i j} \cos \left(j N_{a} \omega_{c} t-j N_{a} \psi_{i}+\theta_{j}\right),
\end{aligned}
$$

where $X_{i j}$ represents the Fourier coefficient of the $j$ th gear mesh harmonic of the vibration signal from the annulus gear and $i$ th planet mesh. $-\psi_{i} / \omega_{c}$ is the time shift between the annulus - $i$ th planet and annulus - 1st planet mesh. $\theta_{j}$ is the initial phase of the $j$ th gear mesh harmonic.

On the other hand, the vibration that resulted from the sun gear and $i$ th planet mesh is presented as $y_{i}(t)$ in (1). Since the vibration signal $y_{i}(t)$ is periodic at the fundamental gear mesh frequency $\omega_{m}$, it can be also written as a Fourier series [11]:

$$
\begin{aligned}
& y_{i}(t)=\sum_{j=1}^{J} Y_{i j} \\
& \cdot \cos \left(j\left(N_{s}\left(\omega_{s}-\omega_{c}\right)\left(t+\frac{\psi_{i}}{\omega_{s}-\omega_{c}}\right)+\gamma\right)+\delta_{j}\right) \\
&=\sum_{j=1}^{J} Y_{i j} \cos \left(j N_{s}\left(\omega_{s}-\omega_{c}\right) t+j N_{s} \psi_{i}+j \gamma+\delta_{j}\right),
\end{aligned}
$$

where $Y_{i j}$ is the Fourier coefficient of the $j$ th gear mesh harmonic of the vibration signal from the sun gear and $i$ th planet mesh. As the sun gear is assumed to rotate clockwise in Figure $1, N_{s} \psi_{i}$ is the phase difference caused by the time shift between the sun - $i$ th planet and sun - 1st planet mesh. $\delta_{j}$ is the initial phase of the $j$ th gear mesh harmonic. Additionally, the phase angle $\gamma$ is the phase difference between the sunplanets meshes and annulus-planets meshes [14]. Under the ideal condition, $X_{i j}=X_{1 j}$ and $Y_{i j}=Y_{1 j}$ as the planet pinions are designed to share the equal load.

In case of a fixed-axis gear pair, the amplitude and phase modulation functions, $a_{j}(t)$ and $b_{j}(t)$, are employed to represent the variations of the vibration spectrum caused by a local gear fault. Similarly, when a local gear fault exists in the planetary gear-set, it would also result in amplitude and phase modulations in addition to the vibrations contributed from sun-planets meshes and annulus-planets meshes as described by (1)-(3). Thus, under the faulty condition, the vibration signal of the annulus - $i$ th planet and sun - $i$ th planet mesh can be further expanded, respectively, [11]:

$$
\begin{aligned}
& x_{i}^{\prime}(t)=\sum_{j=1}^{J} X_{j}\left(1+a_{j}\left(t-\frac{\psi_{i}}{\omega_{c}}\right)\right) \\
& \cdot \cos \left(j N_{a} \omega_{c}\left(t-\frac{\psi_{i}}{\omega_{c}}\right)+b_{j}\left(t-\frac{\psi_{i}}{\omega_{c}}\right)\right),
\end{aligned}
$$

$$
\begin{aligned}
& y_{i}^{\prime}(t)=\sum_{j=1}^{J} Y_{j}\left(1+a_{j}\left(t+\frac{\psi_{i}}{\omega_{s}-\omega_{c}}\right)\right) \\
& \cdot \cos \left(j\left(N_{s}\left(\omega_{s}-\omega_{c}\right)\left(t+\frac{\psi_{i}}{\omega_{s}-\omega_{c}}\right)+\gamma\right)\right. \\
& \left.+b_{j}\left(t+\frac{\psi_{i}}{\omega_{s}-\omega_{c}}\right)\right),
\end{aligned}
$$

where the amplitude and phase modulation functions, $a_{j}(t)$ and $b_{j}(t)$, can be presented:

$$
\begin{aligned}
& a_{j}(t)=\sum_{l=1}^{L} A_{j l} \cos \left(l \times \omega_{\text {characteristic }} t\right), \\
& b_{j}(t)=\sum_{l=1}^{L} B_{j l} \cos \left(l \times \omega_{\text {characteristic }} t\right) .
\end{aligned}
$$

If there is a local fault on the annulus gear, its corresponding characteristic frequency is $\omega_{\text {characteristic }}=\omega_{c}$, which is the rotational frequency of the annulus relative to the carrier. If a damaged tooth exists on the sun gear, it would influence the gear mesh with a frequency that equals the rotational frequency of the sun gear relative to the carrier. Thus, the characteristic frequency caused by the sun gear fault is periodic with a fundamental frequency $\omega_{s}-\omega_{c}$. Analogically, the amplitude and phase modulation functions, $a_{j}(t)$ and $b_{j}(t)$, corresponding to a fault on a planet are periodic with a fundamental frequency that equals the rotational frequency of the planet relative to the carrier, $\omega_{c}+\omega_{p}$. Generally, the analytical model presented by (1)-(5) can predict the distinct behaviors of fault introduced sidebands picked by the fixed accelerometer and its prerequisite input is just the basic geometry of the planetary gear-set. Figure 2 plots the predicted results of this analytical model on the hypothesis of faults occurring at different components of a planetary gearset.

\section{Scheme of the Proposed Diagnostic Algorithm}

A novel vibration based diagnostic scheme is proposed in this section to enable the automated enhancement and identification of fault signatures for planetary gear systems, which is shown in Figure 3. The details of each step are explained as follows.

Step 1. As discussed in the last sections, the vibration signal $v(t)$ contains rich diagnostic information, which is measured by an accelerometer mounted on the stationary annulus gear as shown in Figure 1. Although the behaviors of sidebands are relatively more complex than the cases of fixed-axis gear-sets, many of these sidebands in the frequency domain can still be used as fault signatures to detect and identify the local gear faults. However, in practical applications, the fluctuations of the operational speed can smear the frequency components of the spectrum and the rotary encoders/tachometers are 


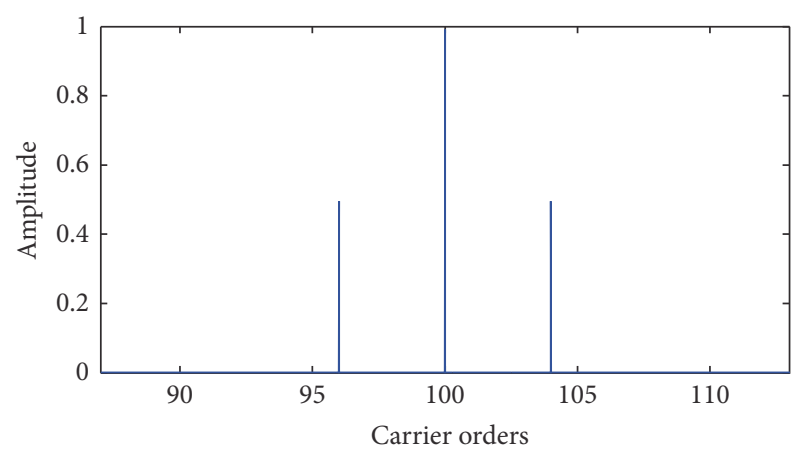

(a)

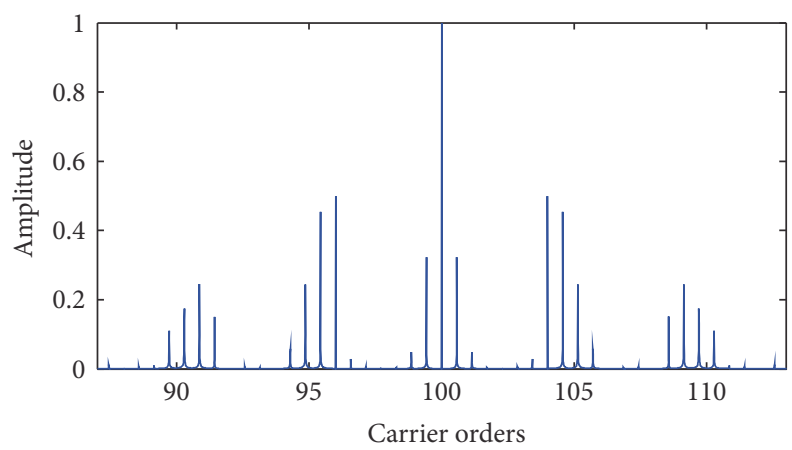

(c)

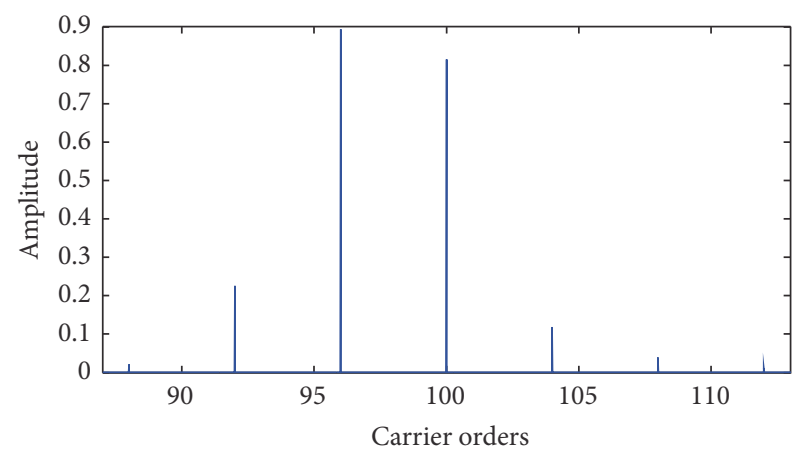

(b)

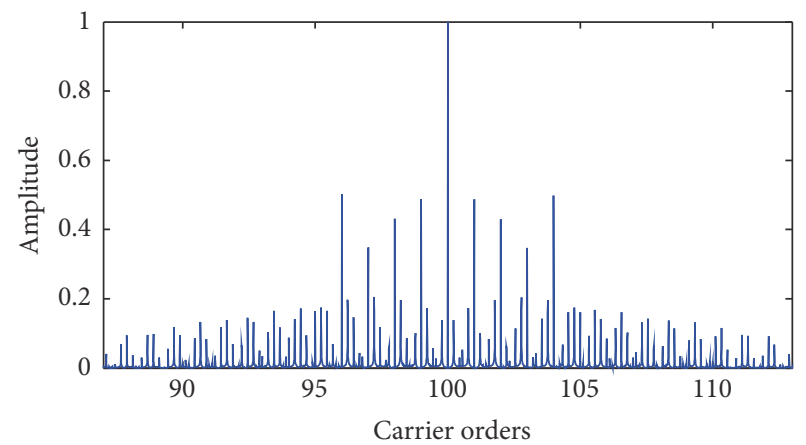

(d)

Figure 2: Predicted vibration spectrum around the fundamental gear mesh frequency picked by a fixed vibration sensor on the annulus for $N_{a}=100, N_{s}=28, N_{p}=36$, and $P=4$; (a) healthy case; (b), (c), and (d) a local gear fault on the annulus, sun, and one planet gear, respectively.

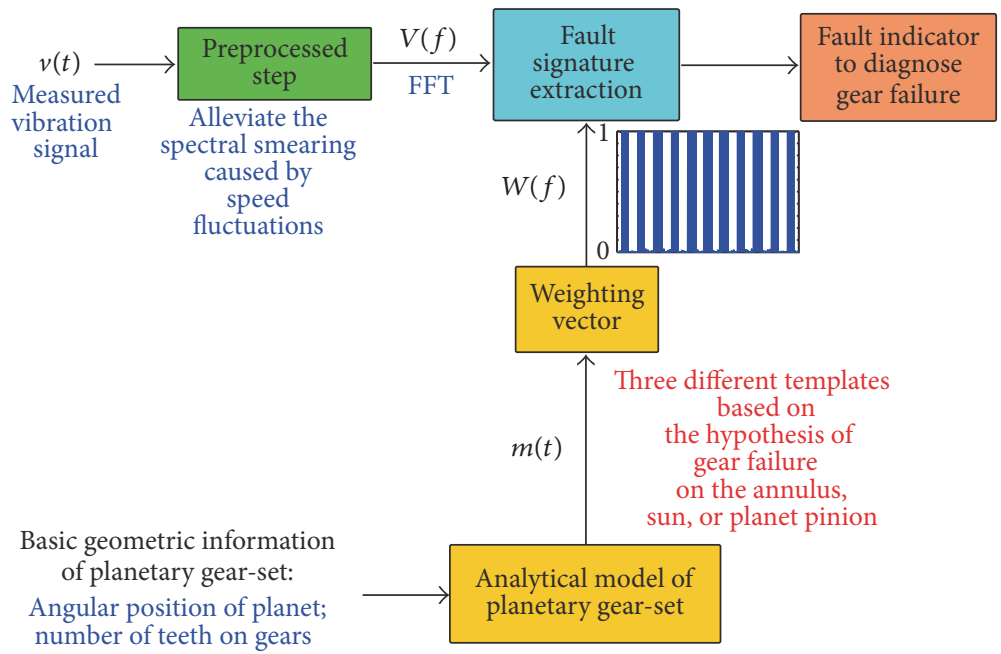

FIGURE 3: The architecture of the proposed vibration based diagnostic scheme.

usually absent in industrial drive trains. Fortunately, tacholess order tracking algorithms have been developed to remove the smearing effect caused by the speed fluctuations, most of which are based on the estimation of the instantaneous frequency and sophisticated interpolations are required to resample the vibration data $[15,16]$. Recently, an original pattern based tacholess algorithm has been proposed by the author to remove the spectral smearing and squeeze the smearing components as individual peaks [17]. After the preprocessing to alleviate the blurred spectrum caused by the speed fluctuations, the resulted vibration signal $\bar{v}(t)$ is then transformed into the frequency domain as the vibration spectrum $V(f)$.

Step 2. To extract the fault introduced information of the profuse sidebands masked in the noisy vibration spectrum 


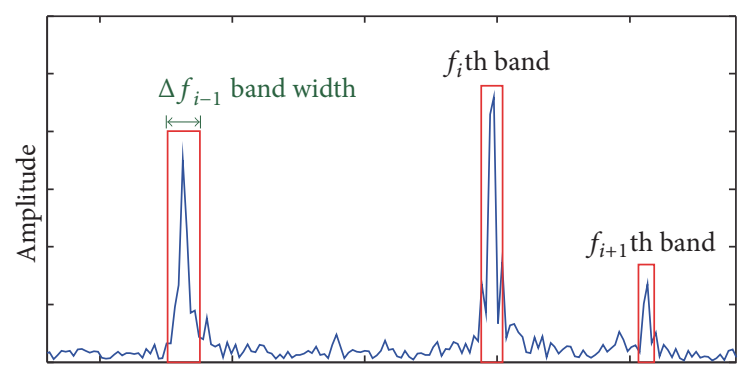

Frequency

FIGURE 4: Illustration of the proposed comb filter.

$V(f)$, a comb filter is specifically designed herein for the planetary gear system based on the analytical model reviewed in the last section. The designed comb filter can be described with two variables vectors: $f$ and $\Delta f$ as presented in Figure 4, where the variable $f_{i}$ represents the center frequency of the $i$ th comb band and another variable $\Delta f_{i}$ defines the corresponding bandwidth of $f_{i}$.

The center frequencies $f$ of the comb filter are chosen as follows: the basic geometric information of the monitored planetary gearbox; that is, the number of teeth on the annulus, sun, and planet pinions, the number of planets, and the angular locations of planets serve as the input to the analytical model as given in (1)-(5). This model is used to generate vibration response templates $m(t)$ that summarize the sidebands characteristics of the healthy case and all the possible fault scenarios. Such model enables the capability of the identification of the gear fault. Then, the frequency components of the spectrum of the template $m(t)$, which have the nonzero magnitudes, are normalized to one to obtain a weighting vector $W(f)$. The normalization is necessary because the practical magnitudes of the frequency components in the measured vibration spectrum rely on the system specification of the gearbox, the operating conditions, and the severity of the faults, which cannot be estimated by the analytical model. These frequency components having the nonzero magnitudes correspond to the center frequencies $f$ of the comb bands.

An important feature for the success of the proposed comb filter is that the bandwidth $\Delta f$ should be selective enough so unwanted noisy components are correctly filtered out from the signal and all the energy of the fault introduced sidebands is kept in the filtered signal. $\Delta f_{i}$ may be relatively small and it is independent of the center frequencies $f$ of the comb bands. In order to automatically determine a proper bandwidth $\Delta f_{i}$ for the $i$ th comb band, statistical indicators are proposed to supervise the exhaustive search. The main principle of the proposed intelligent selective method is illustrated in Figure 5. The main peak plotted in blue color in Figure 5 mimics the $i$ th sideband caused by the gear fault. In practice, the measured vibration signals are usually contaminated by the white noise, which is illustrated in Figure 5(b). The parasite peak highlighted in yellow color in Figure $5(\mathrm{c})$ is used to mimic the noise introduced by the other mechanical component of the gearbox. An arbitrary chosen white noise series is given in Figure 5(d) for reference. It can be observed from Figure 5(c) that when the bandwidth $\Delta f_{i}$ is smaller than the bandwidth of the main energy peak, the kurtosis of the spectrum in the selected frequency band decreases with the increase of the bandwidth $\Delta f_{i}$. However, when the bandwidth is larger than the desired main peak, the kurtosis value begins to increase with the increase of the bandwidth $\Delta f_{i}$. The same phenomenon is also applicable to the scenarios in Figures 5(a) and 5(b). Thus, the value of $\Delta f_{i}$ can be selected according to this watershed as the proper bandwidth for the $i$ th comb band. Generally speaking, a small RMS value of a comb band means that there is just background noise within this comb band as shown in Figure 5(d), the magnitudes of which can be set as zero to filter out the white noise.

Eventually, the obtained frequency lines $f$ of $W(f)$ and their selected bandwidth $\Delta f$ are used to form the comb filter to exclude the noise and extract the fault features. This comb filter allows for an automated highlight and identification of any additional frequency components caused by gear faults, which are located at frequencies depending on the geometry of the planetary gear-set and the location of the local fault.

Step 3. To intelligently diagnose the location of the local fault and the severity of the tooth damage, a frequency domain statistical metric, absolute-value logarithmic ratio (ALR), is designed as the fault indictor, which can be computed over the extracted signal after the denosing process:

$$
\operatorname{ALR}=\frac{1}{N} \sum_{n=1}^{N}\left|\log _{e} \frac{|\alpha(n)|_{\mathrm{af}}}{|\alpha(n)|_{\mathrm{bf}}}\right|,
$$

where $N$ is the number of the extracted nonzero frequency components and $\alpha(n)$ is the magnitude of the $n$th extracted frequency component. The subscripts "af" and "bf" denote after and before the local tooth damage affecting the vibration response, respectively. As manufacturing errors often exist in the practical gearboxes, previous publications found that the occurrence of an incipient damage at a single tooth can cause either the increase or decrease in magnitudes at certain frequency components with almost equal probability $[18,19]$. Because of this, the value of the metric $\log _{e}\left(|\alpha(n)|_{\mathrm{af}} /|\alpha(n)|_{\mathrm{bf}}\right)=\log _{e}\left(|\alpha(n)|_{\mathrm{af}}\right)-\log _{e}\left(|\alpha(n)|_{\mathrm{bf}}\right)$ can be either positive or negative. Thus, the absolute value is proposed in (6) to ensure that the accumulated fractional change $|\alpha(n)|_{\mathrm{af}} /|\alpha(n)|_{\mathrm{bf}}$ can always be regarded as incremental. As a consequence, the proposed fault indictor ALR assesses the changes, either increase or decrease, in the individual magnitudes of the extracted frequency components. The effect of using the natural logarithm in (6) is to substantially underweight the manufacturing errors so that they do not dominate the fault detection indictor in (6) during its real application [19]. Since the magnitudes of fault signatures increase with the fault degradation of the gear tooth, a larger value of the ALR implies a more severe fault [20]. Thus, the ALR is capable of assessing the severity of gear faults. The dynamic analysis of gearbox and experimental pretest can determine the appropriate thresholds of ALR for the classification of the severity of gear faults. In industrial 


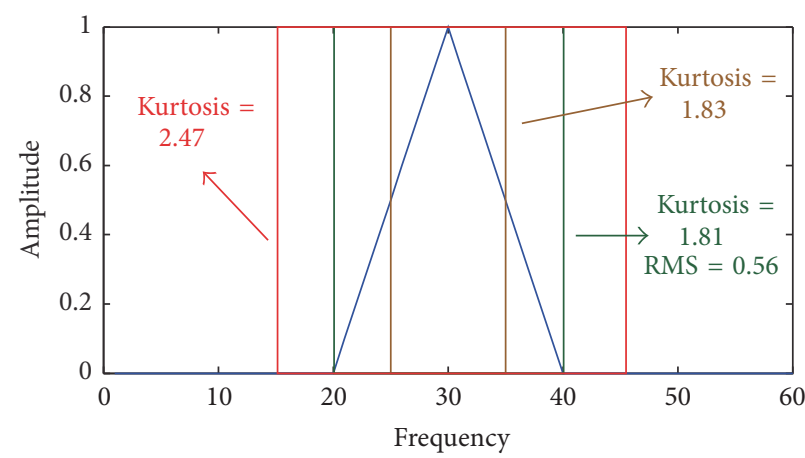

(a)

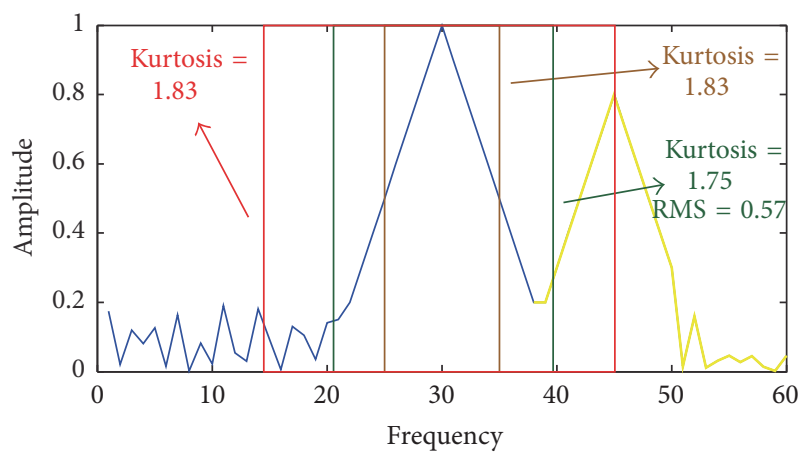

(c)

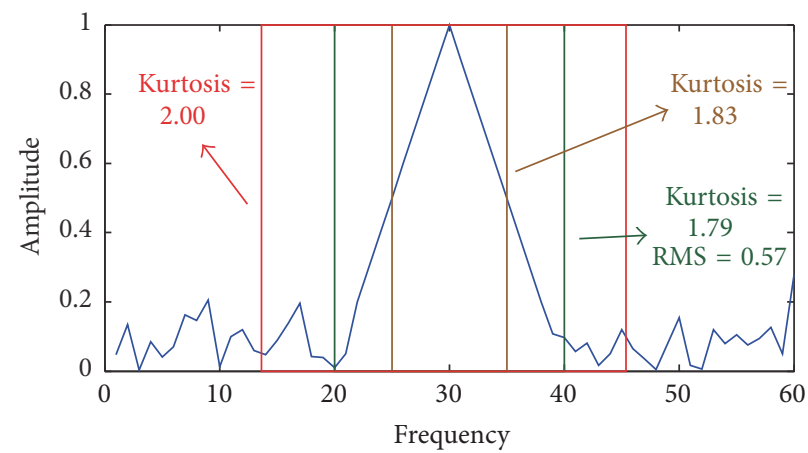

(b)

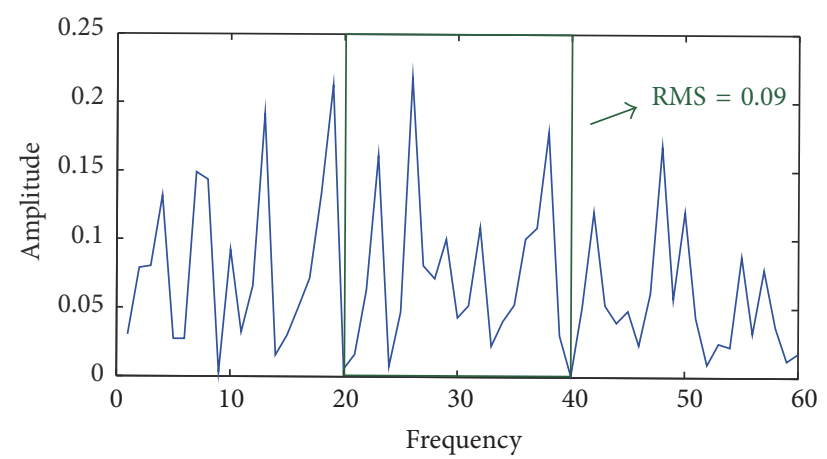

(d)

FIGURE 5: Illustration of the intelligent bandwidth selective principle: (a) single spectral peak; (b) single spectral peak with background noise; (c) single spectral peak with a parasite peak and background noise; (d) background noise.

environments, when the state and system parameters of a gearbox are unavailable, the historical data can be referred as the set of "bf" to track the degradation process of the gearbox. If the value of ALR equals 0 , it implies no change in the individual sidebands and thus means free of fault in the monitored gearbox.

\section{Simulation Results}

4.1. Lumped Parameter Model of Planetary Gearbox. A twostage gearbox is simulated to test the efficacy of the proposed diagnostic scheme. The schematic of this simulated planetary gearbox is illustrated in Figure 6. The first stage is an equally spaced planetary gear-set with 4 planets, in which $N_{a}=100$, $N_{s}=28$, and $N_{p}=36$. The second stage is a fixed-axis gear pair, whose wheel gear has 48 teeth $\left(=N_{\mathrm{wg}}\right)$ and pinion gear has 30 teeth $\left(=N_{\mathrm{pg}}\right)$. The total gear ratio of the planetary gearbox equals $4.57 \times 1.6=7.31$. The rotational speed of the input shaft is set as 913 RPM. The fundamental gear mesh frequency of the planetary gear-set and the fixe-axis gear pair is $1521.8 \mathrm{~Hz}$ and $3338.1 \mathrm{~Hz}$, respectively.

The dynamic model of planetary gearbox used in this work is illustrated in Figure 7 and described in detail in $[10,21]$. The gears are simplified as rigid bodies connected to each other along the line of action through the corresponding gear mesh stiffness and viscous damping [22-24], the model

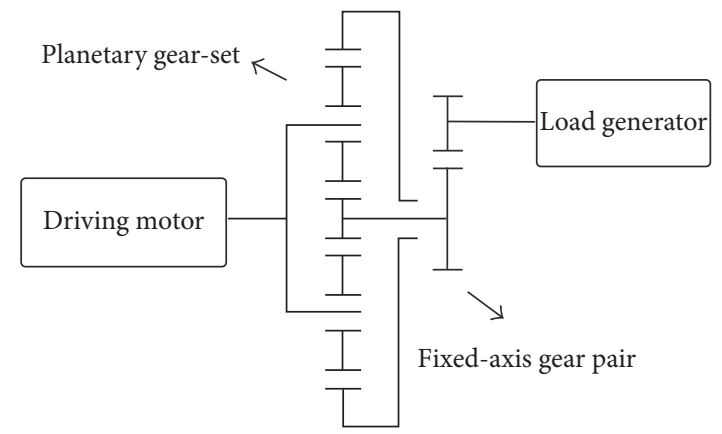

FIgURE 6: Schematic diagram for the simulated planetary gearbox.

of which has been widely applied to study the dynamics of industrial planetary gearbox. Since the gears are held by bearings, such rigid bodies are allowed to translate in $x$ and $y$ directions and to rotate freely around their centers in the $x-y$ transverse plane of gears. Thus, the motion of the sun gear is defined with the translational displacement $x_{s}$ and $y_{s}$ and the angular coordinate $\theta_{s}$. Similarly, the motion of the carrier is defined by $x_{c}, y_{c}$, and $\theta_{c} . \alpha_{i}$ is the pressure angle and $\psi_{i}$ is the initial angle location for planet $i$. The gear imperfections due to the manufacturing errors are simulated to use the error functions $e$ with a $5 \mu \mathrm{m}$ amplitude of saw-tooth [22] as shown in Figure 8(a). Thus, the gear mesh deformation along the line 


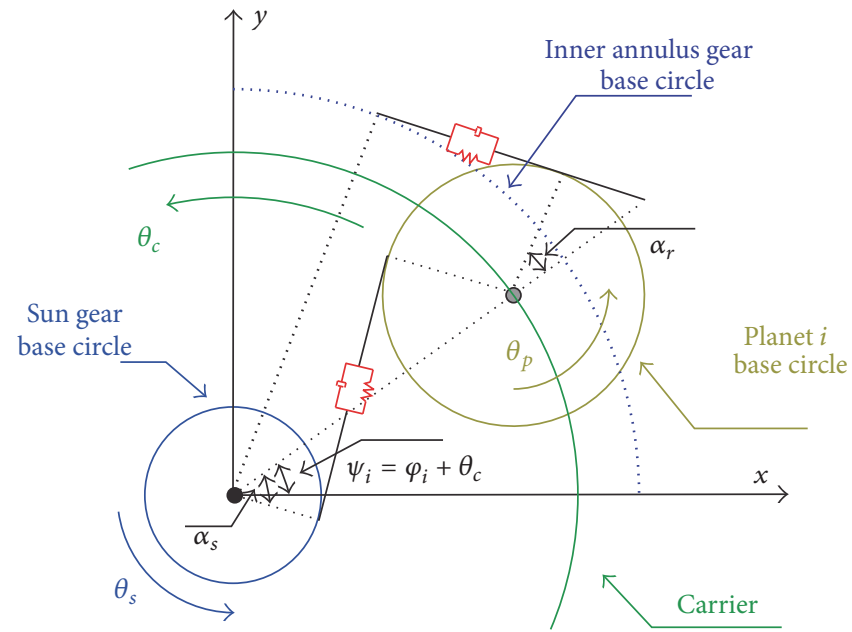

(a)

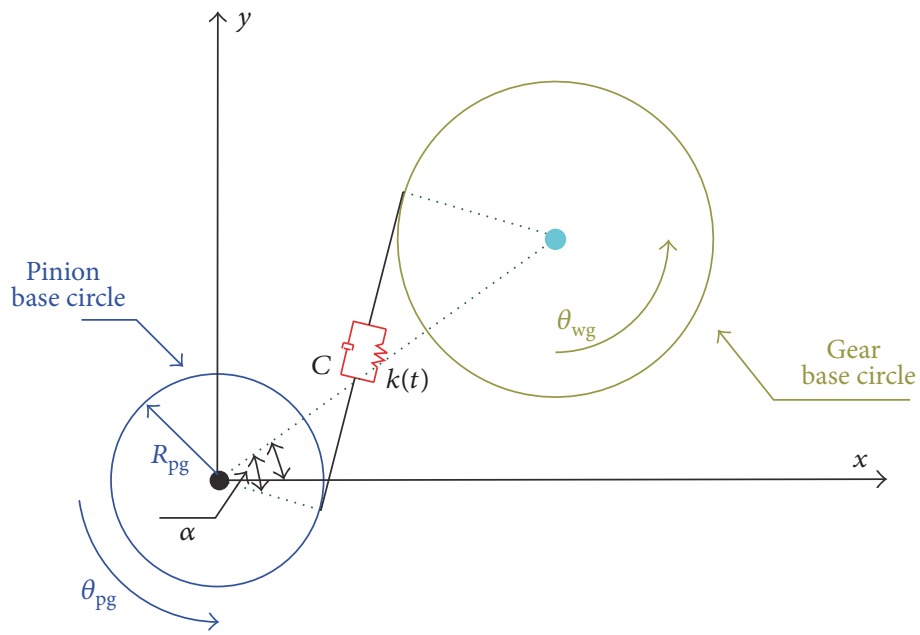

(b)

FIgURE 7: Two-dimensional lumped parameter model of a planetary gearbox: (a) the planetary gear-set and (b) the fixed-axis gear stage.

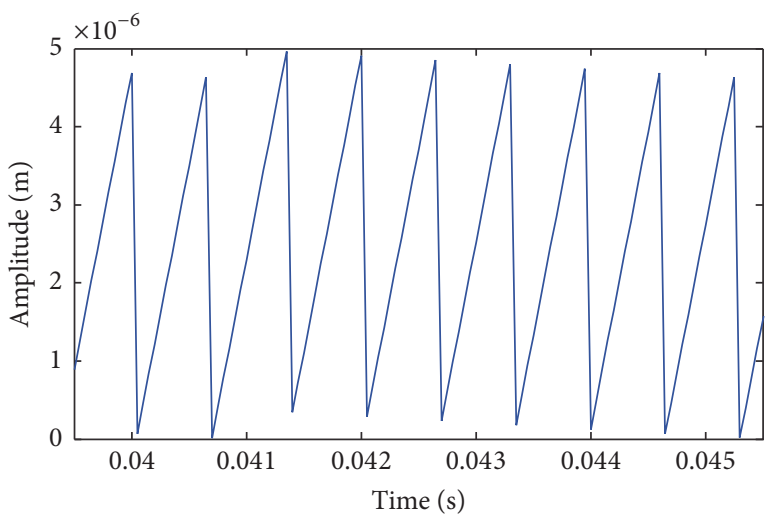

(a)

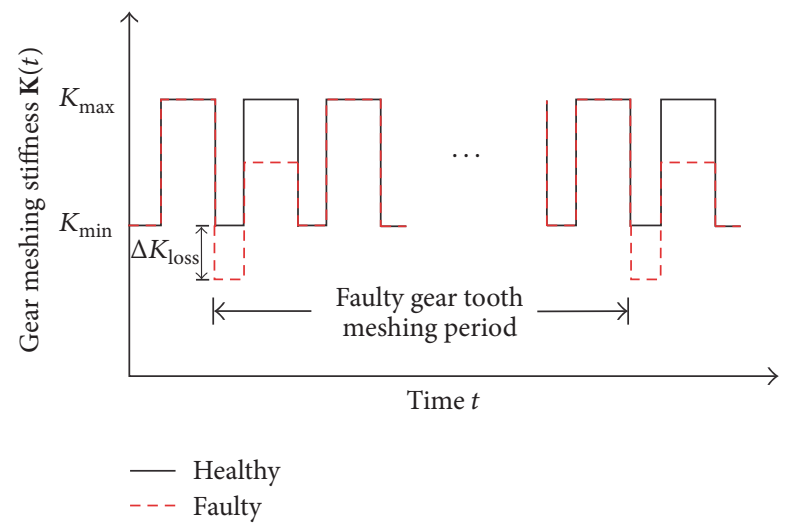

(b)

FIGURE 8: (a) Error function and (b) gear mesh stiffness under the healthy and faulty case. 
of action between the sun gear and the $i$ th planet gear can be defined as ( $R$ is the radius of the base circle):

$$
\begin{aligned}
\delta_{\mathrm{spi}}= & \left(x_{s}-x_{i}+R_{c} \cos \psi_{i}\right) \sin \left(\alpha-\psi_{i}\right) \\
& +\left(y_{s}-y_{i}+R_{c} \sin \psi_{i}\right) \cos \left(\alpha-\psi_{i}\right) \\
& +\left(\theta_{s}-\theta_{c}\right) R_{s}-\left[-\left(\theta_{p}-\theta_{c}\right)\right] R_{p}+e_{\mathrm{spi}}
\end{aligned}
$$

The gear mesh deformation between the annulus gear and the $i$ th planet gear can be written as

$$
\begin{aligned}
\delta_{\mathrm{api}}= & \left(x_{i}-R_{c} \cos \psi_{i}\right) \sin \left(\alpha+\psi_{i}\right) \\
& -\left(y_{i}-R_{c} \sin \psi_{i}\right) \cos \left(\alpha+\psi_{i}\right)-\left(\theta_{i}-\theta_{c}\right) R_{p} \\
& -\left[-\left(0-\theta_{c}\right)\right] R_{r}+e_{\mathrm{api}},
\end{aligned}
$$

where the $i$ th planet pinion has rotation $\theta_{i} . x_{i}$ and $y_{i}$ are the translational displacements of the $i$ th planet.

The gear mesh deformation between the wheel gear and pinion gear along the line of action is expressed as

$$
\begin{aligned}
\delta= & \left(x_{\mathrm{wg}}-x_{\mathrm{pg}}\right) \sin \alpha+\left(y_{\mathrm{wg}}-y_{\mathrm{pg}}\right) \cos \alpha+\theta_{\mathrm{wg}} r_{\mathrm{wg}} \\
& -\theta_{\mathrm{pg}} r_{\mathrm{pg}}+e,
\end{aligned}
$$

where $\theta$ represents the rotational motion. $x$ and $y$ are the translational displacements of the gears. The subscripts wg and pg correspond to the wheel gear and pinion gear of the fixed-axis gear stage, respectively. $R_{\mathrm{wg}}$ and $R_{\mathrm{pg}}$ are the radius of the base circle of the wheel gear and pinion gear. $\alpha$ is the pressure angle of the fixed-axis gear pair.

Then, the dynamic gear mesh forces of the gear pair $F_{m}(t)$ can be further given as

$$
F_{m}(t)=c \dot{\delta}+k(t) \delta
$$

where $c$ is the damping coefficient and $k(t)$ is the time-varying gear mesh stiffness between a gear pair. The square waveform is often used to represent $k(t)$ for spur gear pairs, which is illustrated in Figure $8(\mathrm{~b})$. The maximum and minimum values of gear mesh stiffness are estimated to be $K_{\max }=5 \times$ $10^{8} \mathrm{~N} / \mathrm{m}$ and $K_{\min }=3 \times 10^{8} \mathrm{~N} / \mathrm{m}$ for the simulated planetary gearbox. Furthermore, previous literatures have concluded that the local gear faults are always accompanied by a local reduction in the gear mesh stiffness. Thus, the common local gear faults are modeled by assuming a local drop from the general squared waveform of the gear mesh stiffness. In this work, the tooth damage-induced variation, 35\% and $65 \%$ local stiffness loss $\Delta K_{\text {loss }}$, is used to simulate the moderate and severe local sun gear faulted cases, respectively.

Finally, the global equation of motion for the gearbox can be expressed in the matrix form as

$$
\mathbf{M Q ̈}(t)+\left[\mathbf{C}+\mathbf{C}_{b}\right] \dot{\mathbf{Q}}(t)+\left[\mathbf{K}(t)+\mathbf{K}_{b}\right] \mathbf{Q}(t)=\mathbf{F}(t),
$$

where $\mathbf{M}$ is the mass matrix, $\mathbf{C}$ is the damping matrix, $\mathbf{K}(t)$ is the time-varying gear mesh stiffness matrix, $\mathbf{C}_{b}$ and $\mathbf{K}_{b}$ are the bearing damping and stiffness matrices, $\mathbf{F}(t)$ is the externally applied torques vector, and $\mathbf{Q}(t)$ is the degrees of freedom vector that contains two coordinates for translational vibration and a coordinate for torsional motion for each gear in the plane containing the gear. The dynamic equations of the lumped parameter of gear-sets are numerically integrated in MATLAB/Simulink environment using ode45 solver and a $10 \mathrm{~dB}$ white noise is then superimposed to the results to simulate the background noise of the practical system.

4.2. Fault Detection. Figure 9 plots the simulated vibration spectrums around the second gear mesh harmonic of planetary gear-set $(3043.5 \mathrm{~Hz})$ under the healthy and severe local sun gear tooth faulted conditions. The raw spectrums in Figure 9 are not straightforward to interpret due to the complexities in the vibration response of the planetary gearset itself. The vibration signals from the planetary gear-set are also superimposed with the background noise and the vibration contribution from the fixed-axis gear pair with run-out errors (the gear mesh frequency and its sidebands caused by the run-out error of the fixed-axis gear pair around $3338.1 \mathrm{~Hz}$ ). From such raw spectrum of the multistage planetary gearbox, it is difficult to conclude whether there is any fault in the planetary gear-set or identify the location of the gear failure.

Thus, the developed diagnostic scheme shown in Figure 3 is applied to the simulated data to enhance the fault signatures. Figure 10 presents the processed spectrum with the proposed scheme, the filter template of which is based on the hypothesis of the sun gear fault scenario. The variations at the extracted frequency features in the spectrum caused by the local fault on the sun gear are now more easily visible in Figure 10.

Then, the proposed ALR is calculated using (6) with the spectrum from Figure 10. The calculated ALR of the moderate local sun gear fault case is 0.99 and the ALR of the severe local sun gear fault case equals 1.28, which indicates the capability of ALR to assess the severity of gear failure in the planetary gearbox. On the other hand, the calculated ALR nearly approaches 0 when applying the filter template on the hypothesis of the annulus and planet gear fault scenario. It implies that the examined planetary gear-set is free of the annulus and planet gear fault. Thus, this simulated case also verifies the effectiveness of the proposed scheme to diagnose the location of the gear failure.

In general, a gear tooth fault is always accompanied by a loss of gear mesh stiffness [1]. The reduction of gear mesh stiffness due to the incipient spalling or tooth breakage fault is within $10 \%$ of the original gear mesh stiffness [20]. The moderate spalling or tooth breakage fault can cause around $10 \%$ to $40 \%$ stiffness loss compared with the original gear mesh stiffness. When there is over $40 \%$ loss of the gear mesh stiffness, the tooth damage is labelled as the severe fault. Accordingly, extended simulations as presented in this section are performed to determine the appropriate thresholds of ALR for the assessment of fault severity, in which the simulated gearbox is assumed to have a $10 \%$ and $40 \%$ local stiffness loss, respectively. Based on the simulation results, the thresholds of ALR for the classification of fault severity in this work are suggested as follows: $0<\mathrm{ALR} \leq$ 0.34 implies the existence of incipient gear fault, $0.34<$ ALR 


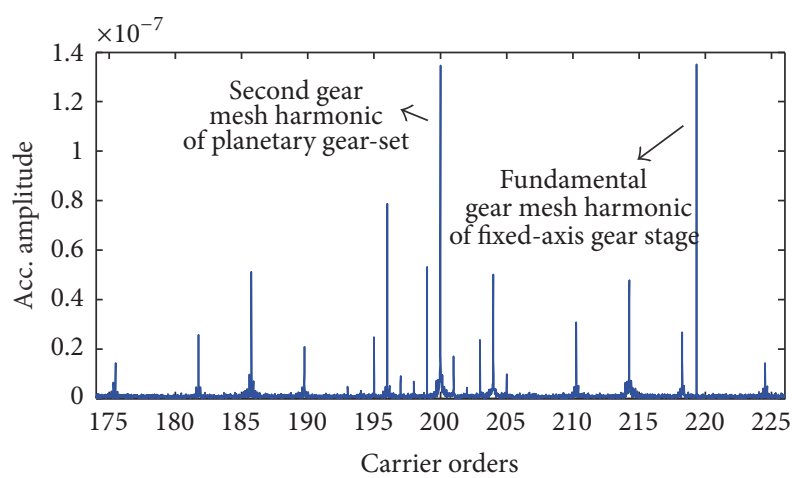

(a)

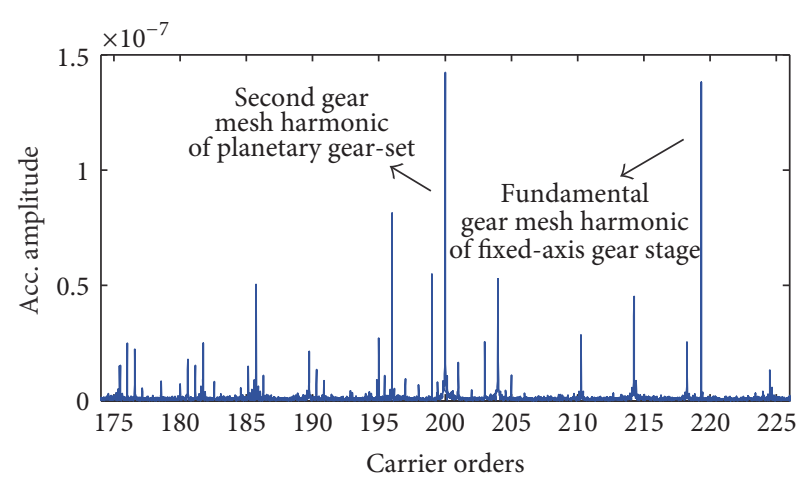

(b)

FIGURE 9: (a) The simulated raw vibration spectrum under the healthy case; (b) the simulated raw vibration spectrum under the severe sun gear faulted case.

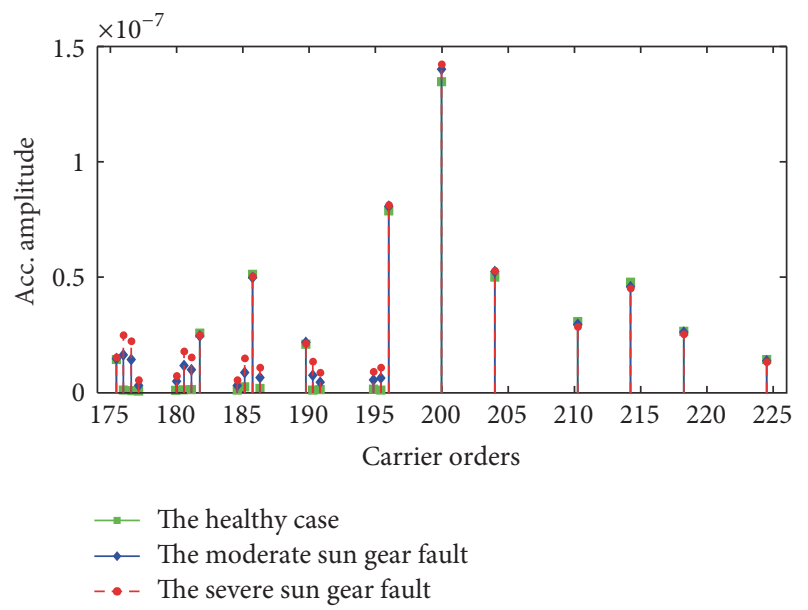

Figure 10: The processed spectrum after the proposed diagnostic scheme.

$<1.09$ indicates the presence of moderate gear fault, and ALR $\geq 1.09$ means there is a severe gear fault. For a specific type of gearbox in practice, the exact threshold boundaries of ALR for the classification of fault severity should be adjusted against the actual system parameters of the gearbox using the proposed dynamic analysis discussed herein. Alternatively, the experiment based approach under typical operational conditions, which uses damaged gears under different stages of fault severity, can also give guideline to determine the thresholds of ALR for the classification of the severity of gear faults.

\section{Experimental Validation}

The proposed vibration based diagnostic scheme was applied to experimentally measured vibration signals from a planetary gearbox test rig shown in Figure 11. The input shaft of the gearbox was connected to a 3-phase AC motor ( $4 \mathrm{~kW} 4$ pole) controlled using an $\mathrm{ABB}$ industrial drive and operated under the nominal speed of 1400 RPM. The load of the drive train was provided by a generator with a resistive load bank, which was connected to the output shaft of the gearbox. The two tested gearboxes were designed as back-to-back planetary gear-sets, each having four equally spaced planet pinions with the number of annulus gear teeth $N_{a}=84$, the number of sun gear teeth $N_{s}=28$, and the number of planet pinion teeth $N_{p}=28$. As the result of the back-to-back configuration, the overall gear ratio of the gearbox equaled $1(4 \times 1 / 4)$. A moderate seeded spalled gear tooth fault was introduced to one of the annulus gear teeth (Figure 11(c)) using the electrodischarge machining (EDM). The vibration signals, plotted in Figure 12, were measured at a sampling rate of $20 \mathrm{KHz}$ using an accelerometer mounted to the gearbox housing outside the annulus gear.

Since the raw vibration signals are often contaminated by the noises and profuse vibration features from other mechanical components of gearboxes, the manual visual investigation of the raw vibration spectrum as shown in Figure 12 is difficult and requires a deep skill and experience that is costly in industrials. Hence, the proposed automated diagnostic scheme for planetary gearbox described in Section 3 is employed to intelligently extract the fault signatures, which correspond to the gear failures in the planetary gear-set. The prerequisite input information of this automated diagnostic scheme is only the number of gear teeth of the monitored planetary gearbox and the approximated operational speed. The extracted fault signatures around the fundamental gear mesh frequency are shown in Figure 13, which is processed by the filter template on the hypothesis of the annulus gear fault scenario. The corresponding calculated ALR equals 0.84 that indicates the existence of a moderate annulus fault in the planetary gearbox. Thus, the effectiveness of proposed diagnostic scheme is validated, which is capable of the fault detection and identification from practical measured signals.

\section{Conclusion}

Failure diagnosis of gearboxes requires high quality fault signatures extracted from the raw measured vibration data to propagate through the fault diagnostic scheme. However, these fault signatures in the raw spectrum are often masked by noises and vibrations of other mechanical components as 


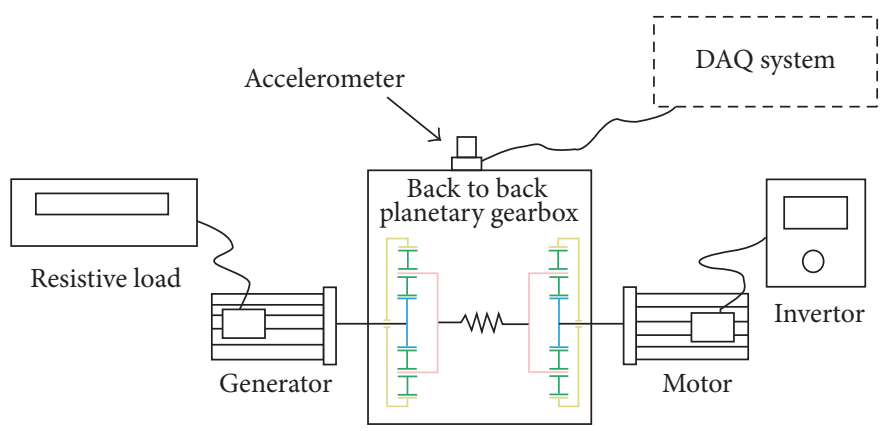

(a)

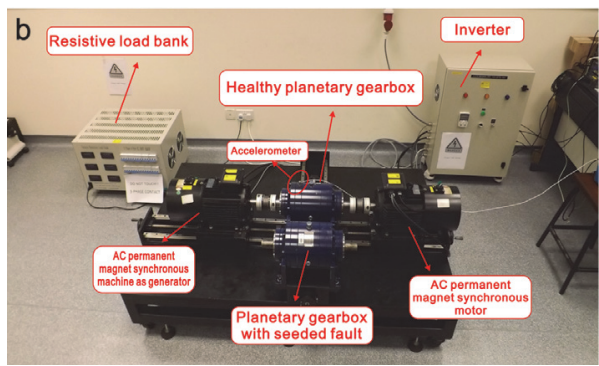

(b)

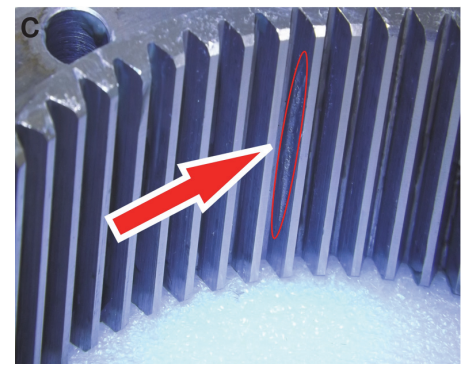

(c)

FIGURE 11: (a) Schematic of the test rig; (b) planetary gearbox test rig; and (c) seeded gear fault on the annulus gear.

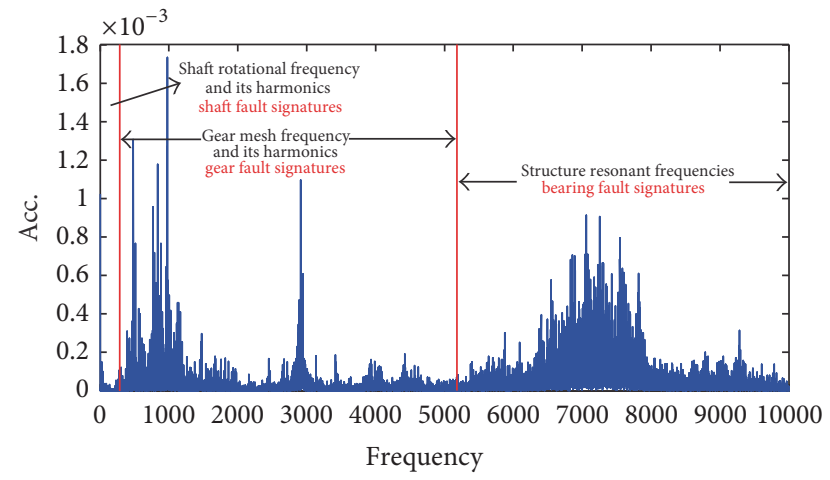

Figure 12: The vibration spectrum of the raw measured signal picked by the fixed accelerometer mounted on the annulus gear.

well as the white noise from the sensors. Due to the complex nature of sidebands in the measured vibration signal picked up by a fixed accelerometer, the application of conventional vibration based diagnostic techniques to planetary gearboxes is a challenge. Hence, this paper develops a novel vibration based diagnostic scheme for planetary gearboxes. The proposed diagnostic system synergistically integrates an analytical model, a denoising algorithm, and frequency domain indicators. This proposed diagnostic scheme can address the limitation of the requirement of extensive stationary data of TSA algorithm, which highlights the fault introduced sidebands by using a comb filter in the frequency domain based on the analytical model of planetary gear-sets. Its performance is firstly tested using simulated signals from a dynamic model of planetary gearbox. Then, the diagnostic

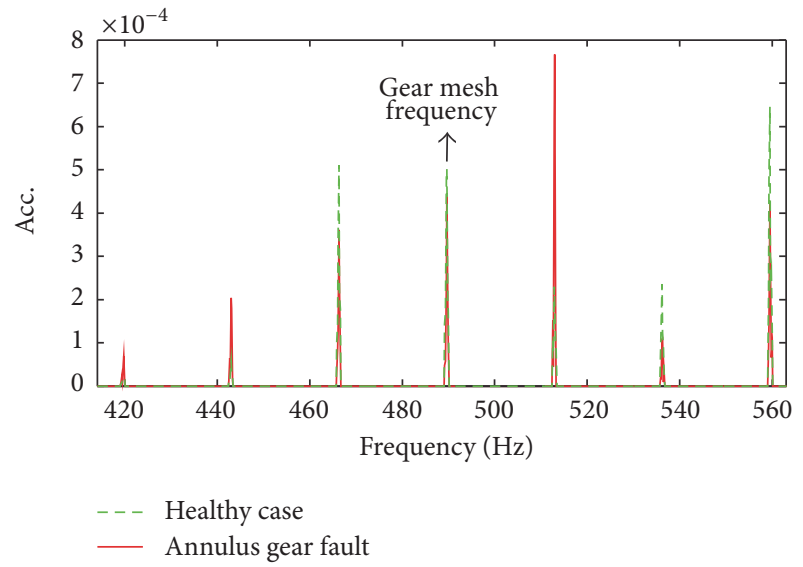

FIGURE 13: The processed spectrum after the diagnostic scheme around the fundamental gear mesh harmonic on the hypothesis of the annulus gear fault scenario.

scheme is also validated with experimental data from a $4 \mathrm{~kW}$ planetary gearbox test rig.

\section{Competing Interests}

The authors declare that they have no competing interests.

\section{Acknowledgments}

The authors are pleased to acknowledge the financial support of National Natural Science Foundation of China (Grant 
no. 51505353) and the Fundamental Research Funds for the Central Universities (WUT: 163104002).

\section{References}

[1] W. Q. Wang, F. Ismail, and M. Farid Golnaraghi, "Assessment of gear damage monitoring techniques using vibration measurements," Mechanical Systems and Signal Processing, vol. 15, no. 5, pp. 905-922, 2001.

[2] J. Urbanek, J. Antoni, and T. Barszcz, "Detection of signal component modulations using modulation intensity distribution," Mechanical Systems and Signal Processing, vol. 28, pp. 399-413, 2012.

[3] P. D. McFadden and J. D. Smith, "An explanation for the asymmetry of the modulation sidebands about the tooth meshing frequency in epicyclic gear vibration," Proceedings of the Institution of Mechanical Engineers. Part C. Mechanical engineering science, vol. 199, no. 1, pp. 65-70, 1985.

[4] W. D. Mark and J. A. Hines, "Stationary transducer response to planetary-gear vibration excitation with non-uniform planet loading," Mechanical Systems and Signal Processing, vol. 23, no. 4, pp. 1366-1381, 2009.

[5] J. McNames, "Fourier series analysis of epicyclic gearbox vibration," ASME Journal of Vibration and Acoustics, vol. 124, no. 1, pp. 150-152, 2002.

[6] M. Inalpolat and A. Kahraman, "A dynamic model to predict modulation sidebands of a planetary gear set having manufacturing errors," Journal of Sound and Vibration, vol. 329, no. 4, pp. 371-393, 2010.

[7] B. Zhang, T. Khawaja, R. Patrick, G. Vachtsevanos, M. E. Orchard, and A. Saxena, "Application of blind deconvolution denoising in failure prognosis," IEEE Transactions on Instrumentation and Measurement, vol. 58, no. 2, pp. 303-310, 2009.

[8] D. G. Lewicki, K. E. LaBerge, R. T. Ehinger, and J. Fetty, "Planetary gearbox fault detection using vibration separation techniques," NASA Technical Report NASA/TM-2011-217127, 2011.

[9] J. M. Ha, B. D. Youn, H. Oh, B. Han, Y. Jung, and J. Park, "Autocorrelation-based time synchronous averaging for condition monitoring of planetary gearboxes in wind turbines," Mechanical Systems and Signal Processing, vol. 70-71, pp. 161-175, 2016.

[10] L. Hong and J. S. Dhupia, "A time domain approach to diagnose gearbox fault based on measured vibration signals," Journal of Sound and Vibration, vol. 333, no. 7, pp. 2164-2180, 2014.

[11] L. Hong, J. S. Dhupia, and S. Sheng, "An explanation of frequency features enabling detection of faults in equallyspaced planetary gearbox," Mechanism and Machine Theory, vol. 73, pp. 169-183, 2014.

[12] Y. Lei, Z. Liu, J. Lin, and F. Lu, "Phenomenological models of vibration signals for condition monitoring and fault diagnosis of epicyclic gearboxes," Journal of Sound and Vibration, vol. 369, pp. 266-281, 2016.

[13] M. Inalpolat and A. Kahraman, "A theoretical and experimental investigation of modulation sidebands of planetary gear sets," Journal of Sound and Vibration, vol. 323, no. 3-5, pp. 677-696, 2009.

[14] R. G. Parker and J. Lin, "Mesh phasing relationships in planetary and epicyclic gears," Journal of Mechanical Design, Transactions of the ASME, vol. 126, no. 2, pp. 365-370, 2004.
[15] F. Bonnardot, M. El Badaoui, R. B. Randall, J. Danière, and F. Guillet, "Use of the acceleration signal of a gearbox in order to perform angular resampling (with limited speed fluctuation)," Mechanical Systems and Signal Processing, vol. 19, no. 4, pp. 766785, 2005.

[16] M. Zhao, J. Lin, X. Wang, Y. Lei, and J. Cao, "A tacho-less order tracking technique for large speed variations," Mechanical Systems and Signal Processing, vol. 40, no. 1, pp. 76-90, 2013.

[17] L. Hong, Y. Qu, J. S. Dhupia, and Y. Tan, "A novel fault diagnostic technique for gearboxes under speed fluctuations without angular speed measurement," in Proceedings of the ASME/ISCIE International Symposium on Flexible Automation, Cleveland, Ohio, USA, August 2016.

[18] L. Hong, Y. Qu, J. S. Dhupia, and Y. Tan, "A novel synergistic diagnostic scheme for planetary gearboxes based on an analytical vibration model of planetary gear-sets," in Proceedings of the Prognostics and System Health Management Conference, Chengdu, China, October 2016.

[19] W. D. Mark, H. Lee, R. Patrick, and J. D. Coker, "A simple frequency-domain algorithm for early detection of damaged gear teeth," Mechanical Systems and Signal Processing, vol. 24, no. 8, pp. 2807-2823, 2010.

[20] F. Chaari, W. Baccar, M. S. Abbes, and M. Haddar, "Effect of spalling or tooth breakage on gearmesh stiffness and dynamic response of a one-stage spur gear transmission," European Journal of Mechanics, A/Solids, vol. 27, no. 4, pp. 691-705, 2008.

[21] W. Kim, J. Y. Lee, and J. Chung, "Dynamic analysis for a planetary gear with time-varying pressure angles and contact ratios," Journal of Sound and Vibration, vol. 331, no. 4, pp. 883901, 2012.

[22] W. Bartelmus, "Mathematical modelling and computer simulations as an aid to gearbox diagnostics," Mechanical Systems and Signal Processing, vol. 15, no. 5, pp. 855-871, 2001.

[23] V. K. Ambarisha and R. G. Parker, "Nonlinear dynamics of planetary gears using analytical and finite element models," Journal of Sound and Vibration, vol. 302, no. 3, pp. 577-595, 2007.

[24] T. M. Ericson and R. G. Parker, "Planetary gear modal vibration experiments and correlation against lumped-parameter and finite element models," Journal of Sound and Vibration, vol. 332, no. 9, pp. 2350-2375, 2013. 


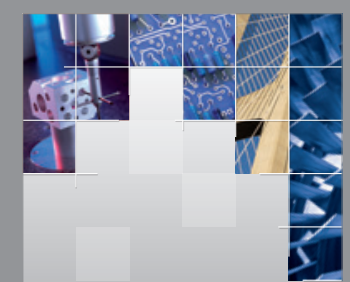

\section{Enfincering}
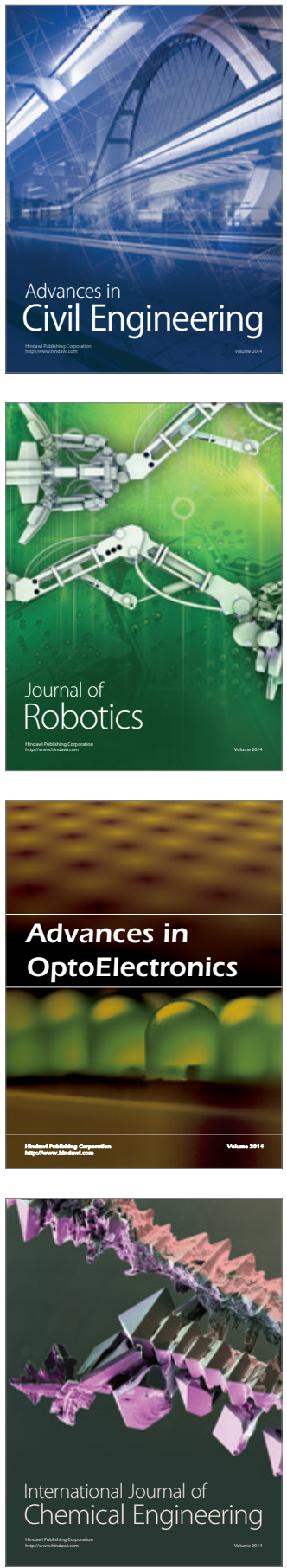

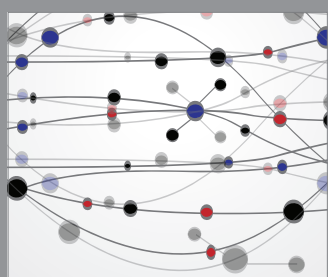

The Scientific World Journal

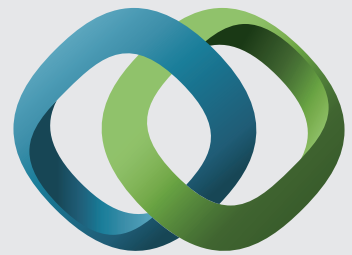

\section{Hindawi}

Submit your manuscripts at

http://www.hindawi.com
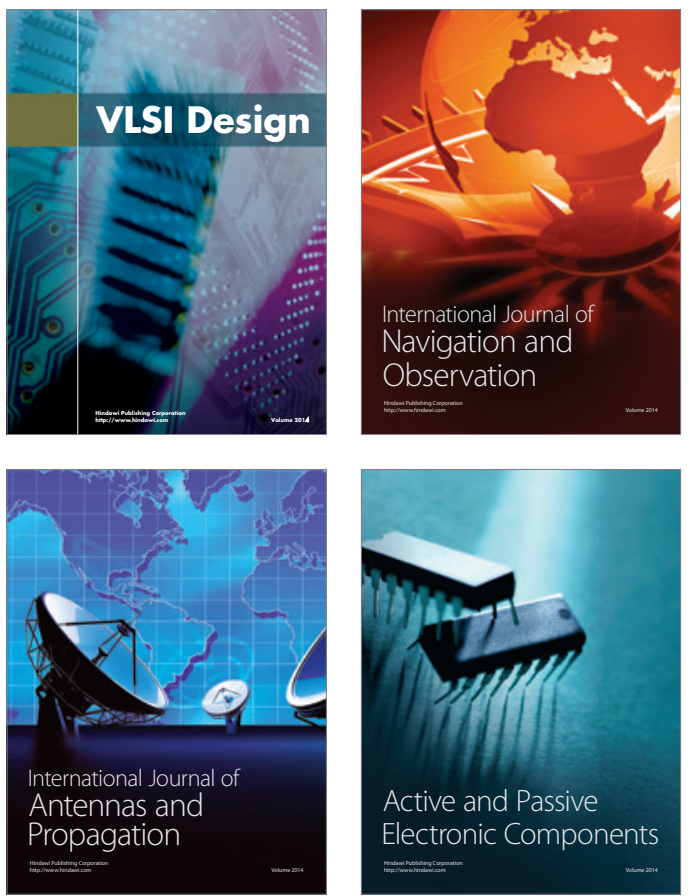
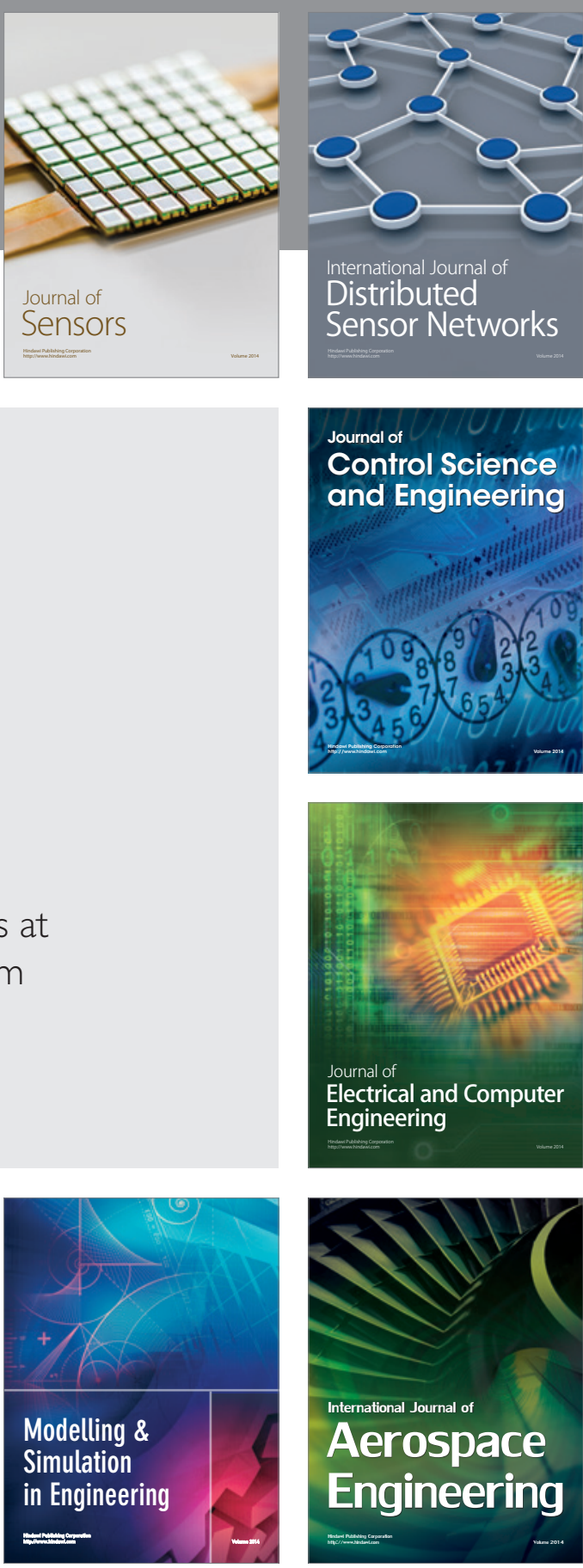

International Journal of

Distributed

Sensor Networks

Journal of

Control Science

and Engineering
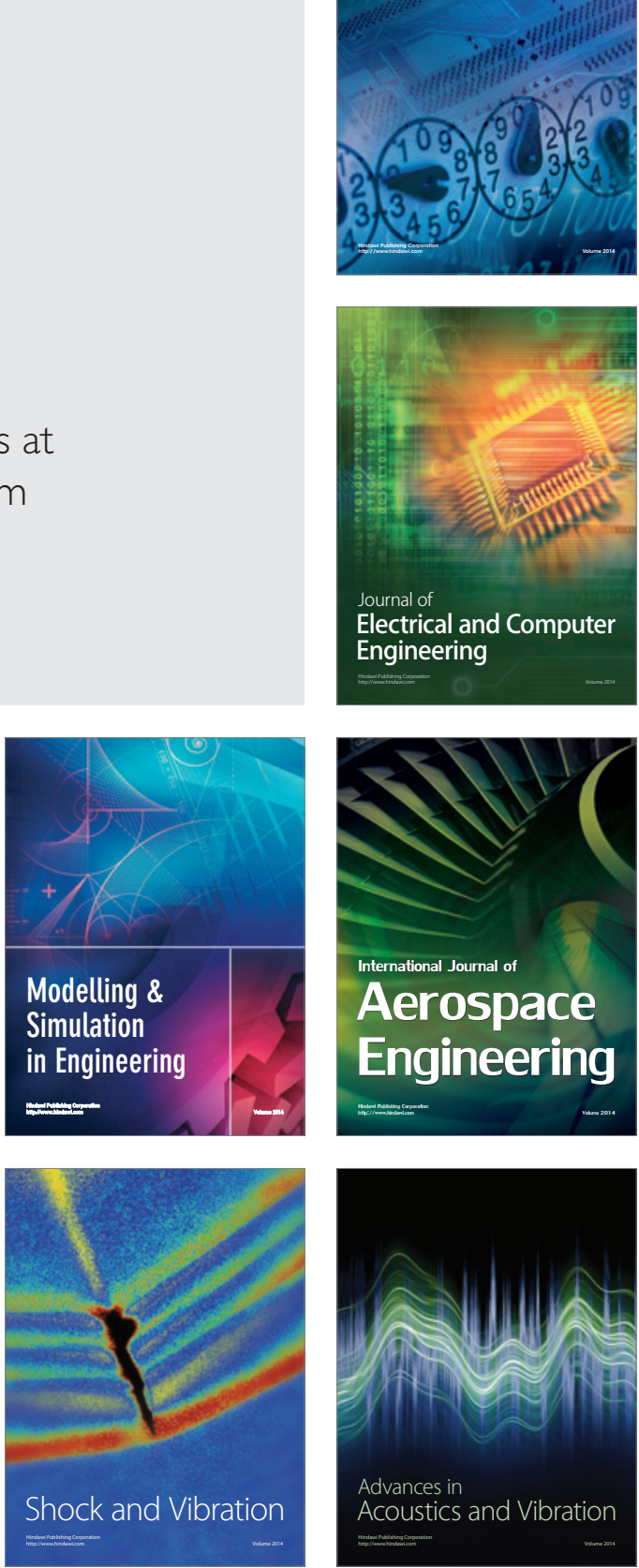